\title{
Heidrun Kämper \& Martin Wengeler (Hg.). 2017. Protest - Parteienschelte - Politikverdrossenheit: Politikkritik in der Demokratie (Sprache - Politik - Gesellschaft 20). Bremen: Dr. Ute Hempen. 266 S.
}

Besprochen von Bettina M. Bock: Universität zu Köln, Institut für deutsche Sprache und Literatur II, Gronewaldstraße 2, D-50931 Köln, E-Mail: bettina.bock@uni-koeln.de

https://doi.org/10.1515/zrs-2018-0008

Der Sammelband widmet sich in einer bemerkenswerten Breite an theoretischen, methodischen und empirischen Zugängen und am Beispiel unterschiedlicher Protestfälle und Kritikformen einem hochaktuellen Thema: Politikkritik und Politikverdrossenheit in der Demokratie. Er geht zurück auf eine Tagung der AG „Sprache in der Politik“ im Mai 2015 in Trier. Auch wenn dies vielleicht zunächst am naheliegendsten erscheint, behandeln die Beiträge gerade nicht nur aktuelle, rechte Protestbewegungen (wie Pegida) oder Politikkritik und Parteienschelte aus neuen rechten Parteien (wie der AfD). Vielmehr wird eine große Vielfalt an Erscheinungsformen von Politikkritik in den Blick genommen. So werden auch linke Protest- und Politikkritikformen interdisziplinär erörtert, die Fallbeispiele sind nicht auf Deutschland beschränkt. Eine Stärke des Bandes liegt v.a. in der Berücksichtigung eines diachronen Blickwinkels. Heidrun Kämper und Martin Wengeler beschreiben die Ausrichtung des Bandes einleitend mit folgenden Worten:

„Artikulation von Unzufriedenheit mit den jeweils existierenden politischen Systemen (der parlamentarischen Demokratie, der Diktatur) ist sowohl in der diachronen als auch synchronen Dimension ein internationales gesellschaftliches Phänomen mit semiotisch komplexen Erscheinungsformen.“ (S. 2)

Während der Gegenstand des Bandes politik- und sozialwissenschaftlich bereits gut bearbeitet ist, so ist er „als politolinguistisches bzw. semiotisches Thema“ eher „unentdeckt“ (ebd.). Anliegen und Anspruch des Bandes ist es, die bisherigen Forschungsarbeiten in diesem Bereich interdisziplinär - insbesondere sprach-, literatur- und kommunikationswissenschaftlich - zu vervollständigen. Aufgegriffen werden in den Beiträgen eine ganze Reihe derjenigen Aspekte, die Kämper in ihrem linguistischen „Klassifizierungsversuch“ von Politikkritik aufführt (S. 20f.): Politikkritik sei auch eine Kommunikationsform, und somit stelle sich die „Frage nach was, wie und wer [...], nach Inhalt, Ausdruck und Akteuren“ (S. 20). Die Beiträge behandeln und analysieren dann sprachliche Ausdrucksund Kommunikationsformen von Politikkritik unter dem Aspekt der verwendeten semiotischen Ressourcen, der Gegenstände von Kritik sowie verschiedener Akteure (Äußerungen von Bürgern, Parteien, Politikern). 
Die drei Beiträge des ersten Abschnitts widmen sich historischen Perspektiven. Heidrun Kämper leistet hier grundlegende Definitions- und Systematisierungsarbeit, indem sie aus historischen Beispielen - sie orientiert sich v.a. an den gesellschaftlichen Umbruchsituationen 1918/19, 1945, 1967/68 - linguistisch perspektivierte Merkmale von Politikkritik ableitet. Am Ende ihres Beitrags liefert sie folgende Definition:

„Politikkritik ist ein komplexes semiotisches und sprachlich-kommunikatives Phänomen, dessen Erscheinung durch historischen Kontext, Gegenstand, Ausdrucksformen und Akteure geprägt ist. Gegenstand von Politikkritik sind politisch-gesellschaftliche Institutionen, politische Akteure oder auf Gesellschaft bezogene Handlungen, die auf unterschiedlichsten Ebenen der Semiotik durch professionell-politische und/oder nichtprofessionell-politische Akteure artikuliert wird.“ (S. 22)

Der Gang durch die historischen Beispiele und die systematische Ableitung von Merkmalen von Politikkritik ist erhellend und instruktiv. Gleichwohl hätte man sich für einzelne Analysen ausführlichere Belege und eine genauere, differenziertere Betrachtung der historischen Fälle gewünscht. Irritierend ist beispielsweise, dass unter der Überschrift „[n]achkriegsdeutsche Ost-West-Kritik“ (S. 15) Politikund v.a. Systemkritik in den beiden Teilen Deutschlands nebeneinander gestellt werden, ohne auf die unterschiedlichen Äußerungs- und Gesellschaftsbedingungen genauer einzugehen. Es wird nahegelegt, dass es sich in beiden Kontexten um dieselben Strategien von Kritik unter gleichen Bedingungen handle. So bezieht sich Kämper auf die „Bekenntniswörter Freiheit, Frieden und Demokratie“ (S. 16), die in Ost und West gleichermaßen für die Kritik des jeweils anderen Systems dienten:

„[I]n Ost wie West dienen die Grundkategorien der politischen Kultivierung, der Etablierung und Konsolidierung des neuen Staats mit der Referenz auf Werte, die zur lexikalischen Instrumentierung des politischen Diskurses spätestens seit 1789 zählen.“ (S. 17)

Hilfreich wäre hier ein zumindest knapper Verweis auf die Unterschiedlichkeit der mit den „Bekenntniswörtern“ verbundenen Bedeutungen sowie auf Unterschiede in der Handhabung und damit der Unterschiedlichkeit der Kritik-Standpunkte und -Bedingungen: einerseits pluralistische Gesellschaft, in der auch Kritik an Demokratie möglich ist, - andererseits Diktatur, in der das Bekenntnis Pflicht ist und Abweichung zu Ausgrenzung führt. Dass in der ideologisch geprägten Systemkritik in Ost und West strukturelle und rhetorische Ähnlichkeiten $\mathrm{zu}$ finden sind, ist kaum zu bestreiten. Dass es Kämper v.a. um eine Strukturierung des Phänomens Politikritik geht, leuchtet ebenfalls ein. Dennoch bleibt auch auf dieser systematischen Ebene eine Leerstelle. Ob und inwiefern in der gleichen historischen Periode (nach 1945) unterschiedliche gesellschaftliche 
Bedingungen (DDR vs. BRD) mit unterschiedlichen Ausdrucksformen von Politikkritik einhergehen, wird nicht thematisiert. Zum Ausdruck kommt dies auch in der m.E. problematischen und unangemessenen unkommentierten Verwendung der DDR-Neuschöpfung ,Volksdemokratie‘ zur (vermeintlich) neutralen Bezeichnung des Regierungssystems der DDR: „Auf die Demokratie bundesrepublikanischer Prägung bzw. die Volksdemokratie der DDR erheben die jeweiligen Protagonisten alleinigen Anspruch und verwerfen das jeweils andere System.“ (S. 21) Eine differenziertere Einordnung der Kritikformen in Ost und West hätte der Definition des Gegenstands sicherlich genützt und hätte zudem den in der Einleitung kurz genannten Gedanken, dass Politikkritik eben nicht nur in Demokratien vorkommt (vgl. S. 2), weiter ausgeführt - was so nun im Band unterbleibt.

Ruth Mell nähert sich dem politikkritischen Konzept der (Gegen-)Öffentlichkeit diskurslexikografisch und stellt die Konzeptualisierungen aus der Zeit um 1968 denen in aktuellen Diskursen gegenüber. Trotz deutlicher Unterschiede gibt es Ähnlichkeiten, denn in aktuellen politik- und medienkritischen Diskursen wird das Konzept der Gegenöffentlichkeit genutzt, um sich gegen die (vermeintlich) manipulativen Massenmedien zu positionieren und „Aufklärung“ über die wahren Verhältnisse zu fordern. Gegenöffentlichkeit wird in diesen Diskursen als „Voraussetzung für Demokratie“ und als „demokratische Gegenmacht“ (S. 36) dargestellt.

Der einzige literaturwissenschaftliche Beitrag des Bandes hinterfragt die These des unpolitischen Charakters von Ernst Jüngers Spätwerk. Wolfgang M. Sснміт zeigt eindrücklich, wie sich trotz der Abwesenheit eines stark militaristischen Sprachgebrauchs die Sprache Jüngers dennoch „bewusst einem demokratischen Diskurs“ entzieht (S. 43). Neben den - vereinzelt doch vorhandenen radikalen, explizit politischen Aussagen, sind es u.a. die Naturschilderungen, die den Autor entlarven: „[S]elbst die Käfer treten [...] als Souveräne auf, sie sind Tyrannen, Führer, Prinzipale, und die Tierchen sollen auf politische und gesellschaftliche Zusammenhänge verweisen“ (S. 45).

Der zweite Abschnitt ist der umfangreichste des Bandes, er widmet sich den kommunikativen Praktiken und sprachlichen Strategien aktueller Politikkritik. Bemerkenswert ist die thematische Stringenz in diesem Abschnitt. Die Beiträge leiten fast „natürlich“ zueinander über. Steffen Papperts Beitrag geht auf eine (oft) kreative, spielerische Form von Politikkritik ein: die Verfremdung von Wahlplakaten, sog. Plakatbusting, das er als „Gegendiskurs zur herrschenden Wahlkampfrhetorik“ (S. 73) und damit als Politikkritik interpretiert. Der Beitrag enthält einen Vorschlag zur Systematisierung von Praktiken der Plakatmodifikation sowie der verwendeten Zeichen und kritisierten Gegenstände. Neben Charlotte SeIler Bryllas Beitrag ist dies damit einer von zwei Beiträgen, der auch nichtsprachliche Formen von Politikkritik - hier: in Bildern - in den Blick nimmt. Auch 
wenn der räumliche Kontext oft unmittelbar bedeutungsrelevant für die Verfremdungen ist, weist Pappert darauf hin, dass es sich beim Plakatbusting durch die mediale (Weiter-)Verbreitung der Plakate um eine Konstellation handle, die nicht nur lokal wirke, sondern ,die der Politikaneignung in den Sozialen Medien vergleichbar“ sei (S. 73). Um soziale Medien geht es dann im darauffolgenden Beitrag von Kersten Sven Roth, der gerade auf die Situationsunabhängigkeit und potenzielle Allgegenwärtigkeit einer bestimmten Form des Sprechens über Politik abhebt. Er untersucht Merkmale von (pauschaler) Politiker-Kritik in Forendiskussionen und stellt fest, dass es hier gerade nicht bestimmte Situationsbedingungen oder bestimmte Sprechergruppen und Räume sind, die die Form der Kritik prägen. Vielmehr sei es eine bestimmte, potenziell allgegenwärtige „Interaktionsmodalität“, die diese Form von Politikkritik kennzeichne (S. 80): Parallelen zieht er zur (analogen) Stammtischkommunikation, bei der ein bestimmter „Modus der sprachlichen Auseinandersetzung mit Politik“ (S. 80) gepflegt wird, der sich vergleichbar auch in den Online-Forendiskussionen belegen lässt: „Es spricht vieles dafür, dass er [der Stammtisch-Modus, B. B.] und mit ihm der typische Sektor der pauschalen Politiker-Kritik anthropologische Grundkonstanten sind.“ (S. 96)

Ebenfalls mit der Kritik von Politikern - diesmal seitens der Medien - befasst sich Melani Schröter. Ihr Gegenstand ist die „Metaberichterstattung“ im britischen Wahlkampf 2015. Gemeint ist damit eine mediale Berichterstattung, die lediglich die Selbstdarstellung und Imagearbeit der Politiker oder die Medienformate und -akteure sowie die Wirkung der medial inszenierten Kommunikationsereignisse beim Publikum thematisiert, aber nicht die inhaltliche Argumentation der Politiker (vgl. S. 99). Die Medien agieren selbstreferenziell und depolitisiert, indem sie dem, was Politiker in Fernsehduellen äußern, pauschal Relevanz und politische Gültigkeit absprechen. Der Beitrag geht damit auf die problematische Rolle der Medien im Diskurs um Politikverdrossenheit und Politikkritik ein. Schröter diagnostiziert für den britischen Kontext eine „Selbstüberhöhung der Medien“ (S. 115). Das Vakuum, „das durch den Fokus auf Inszenierung und Selbstdarstellung politischer Akteure entstanden ist“, füllten auch die Medien nicht (ebd.).

Den zweiten Teil dieses dritten Abschnitts bildet ein Block aus vier Beiträgen zu Straßenprotestformen (Olga Galanova, Mark Dang-Anh, Julia Zilles \& Wolf J. Schünemann, Carolin Waegner). Alle behandeln aktuelle Fälle (u.a. Pegida, Stuttgart 21), die Perspektiven sind soziologisch oder linguistisch. Während GALANOVA \& DANG-ANH eher auf eine theoretische Beschreibung protesthafter Handlungen und deren Einbettung in soziale Situationen bzw. auf die „interaktionale Konstitution einer synthetischen Protestsituation“ in digitalen Medien (S. 133) fokussieren, liegt der Schwerpunkt bei Zilles \& SchünEmann sowie WAEgneR stärker auf der empirischen Analyse der konkreten Protestbeispiele. Die Perspekti- 
ven der einzelnen Beiträge greifen teilweise unmittelbar ineinander. Während Galanova mit einer Betrachtung der Protesthandlungen auf den Straßen beginnt, schließt der Beitrag von Dang-Anh mit der Analyse von Twitter-Interaktionen während einer Pegida-Demonstration an und fragt nach der wechselseitigen Situierung von Straßenprotestsituation und synthetischer Situation durch digitale Kommunikation.

Der dritte Abschnitt des Sammelbandes ist internationalen Perspektiven auf Politikkritik gewidmet. Georg Weidacher betrachtet Fälle aus Österreich und Deutschland; Charlotte SeIler Brylla analysiert grüne Politikkritik in Schweden und Deutschland kontrastiv; JEns MAEßE untersucht aus sozialwissenschaftlicher Sicht das Zusammenspiel von europäischem Krisendiskurs und Parteienkritik und stellt dazu ökonomische Expertendiskurse in Europa gegenüber (S. 247), um dann verschiedene Hegemonien von Krisenakteuren zu beschreiben.

Die linguistischen Beiträge von Seiler Brylla \& Weidacher gehen auf Politikkritik ,aus Innensicht“ ein, nämlich auf von Parteien ausgehende Politikkritik. Im Mittelpunkt stehen bei Seiler Brylla die Anfangsphasen der grünen Parteien in den 1980er Jahren in Schweden und Deutschland, bei Weidacher heutige rechte und linke ,neue Parteien“ (AfD und Piraten in Deutschland, NEOS und Team Stronach in Österreich). Beide Autoren kommen anhand ihrer Analysen zu einer Beschreibung von Ambivalenzen. Einerseits werden etablierte politische und kommunikative Praktiken von den neuen Parteien kritisiert, sie bemühen sich folgerichtig sprachlich und rhetorisch um Abgrenzung. Andererseits streben sie selbst parlamentarischen Einfluss an und bleiben nicht beim Status einer Protestbewegung, sondern kultivieren ihre kritische Grundhaltung aus der eigentlich im ,Establishment' verorteten politischen Form einer Partei heraus. Diese Ambivalenzen schlagen sich in den untersuchten Fällen auch sprachlich-rhetorisch musterhaft nieder, u.a. in Wortoppositionen (alt - neu, die da oben - wir hier unten, Fahnenwort der ,Alternative').

Ein wenig schade ist, dass im Band ein Abschnitt mit „internationalen Perspektiven“ aufgemacht wird, der eben lediglich das Internationale der Gegenstände hervorhebt. Dadurch rücken die systematischen Bezüge zu den anderen Beiträgen m.E. zu Unrecht in den Hintergrund. So, wie der Beitrag von Schröter thematisch eingegliedert wurde, hätte man sich dies auch für die anderen „internationalen“ Artikel gewünscht. Beispielsweise hätte WeIDAchers Unterscheidung zwischen internen (von Parteien ausgehenden) und externen Kritikformen, die auch Kämper aufmacht (S. 20), durchaus als Gliederungsaspekt für den Band genutzt werden können.

Insgesamt bietet der Band in seiner Themen- und Zugangsbreite viele Anregungen und Anknüpfungspunkte für die zukünftige linguistische Forschung im Feld. Das Thema lag ,auf der Straße' und ist nun aufgehoben und mit diesem 
Band in großer Vielfalt und konzentriert an einem Ort versammelt worden. Die Analysezugänge und Methoden sind im Feld der Politolinguistik natürlich nicht völlig neu, aber durch die unterschiedlichen, oft komplementären Perspektiven auf den Gegenstand ergeben sich immer wieder neue, relevante Akzentuierungen. 